\title{
Iron Maiden: la música que gritó cine.
}

Dr. David Fuentefría Rodríguez (Universidad de La Laguna).dfuentef@ull.es

Resumen: Quienes no han seguido la trayectoria musical del grupo británico Iron Maiden ignoran que, además de los grandes hitos de la literatura, las ficciones realistas o metafísicas y los sucesos históricos de alcance, el cine siempre fue una fuente capital de inspiración para sus letras. Encuadrada en la variante de la New Wave of British Heavy Metal (N.W.O.B.H.M.), surgida en el Reino Unido a finales de los 70, la banda cumplirá en 2020 cuarenta años incluyendo en sus discos lo que, por su elevado nivel de creatividad y producción, podrían calificarse como auténticas "bandas sonoras alternativas", que hasta nuestros días siguen contribuyendo a acercar el cine a las nuevas generaciones, mediante la traslación a sus notas, y la novelización/versificación breve, de clásicos y no tan clásicos de toda época, nacionalidad, condición y género, desde "El nombre de la rosa" a "Braveheart", desde "En busca del fuego" a "Planeta prohibido". Este trabajo trata de recopilar, y de poner en valor, todas las canciones del grupo inspiradas en películas, desde su primer disco en estudio, el homónimo "Iron Maiden" (1980), al último y más reciente, "The book of souls" (2015).

Palabras clave: Iron Maiden; música; cine; heavy metal; bandas sonoras; adaptaciones.

Abstract: Those who have not followed the musical trajectory of the British group Iron Maiden ignore that, in addition to the great milestones of literature, 
realistic or metaphysical fictions and historical events of scope, cinema was always a capital source of inspiration for their lyrics. Framed in the variant of the New Wave of British Heavy Metal (NWOBHM), which emerged in the United Kingdom at the end of the 70s, the band will turn in 2020 forty years including in its albums what, due to its high level of creativity and production, they could be described as authentic "alternative soundtracks," which to this day continue to contribute to bringing cinema to the new generations, through the translation to their notes, and the brief novelization / versification, of classics and not so classics of all times, nationality, condition and gender, from "The name of the rose" to "Braveheart", from "Quest for fire" to "Forbidden planet". This work tries to compile, and to put in value, all the songs of the group inspired by films, from their first record, the homonym "Iron Maiden" (1980), to the last and most recent, "The book of souls" (2015).

Keywords: Iron Maiden; music; movies; heavy metal; soundtracks; adaptations.

\section{Introducción. Iron Maiden y la NWOBHM.}

Cuarenta años cumplirá en 2020, sobre los escenarios de todo el mundo, el grupo musical británico Iron Maiden (Doncella de Hierro). A la espera de una biografía definitiva, que llegará con un retiro que se barrunta aún lejano en el tiempo, loas y menciones a la trayectoria de los principales abanderados de la New Wave of British Heavy Metal (NWOBHM) se extienden por todo tipo de estudios y manuales sobre la historia del Rock, amén de constar, merced al juicio del tiempo, en decenas de semblanzas parciales y análisis musicológicos, mientras que sus textos, e incluso su imaginería característica, han sido objeto de investigación en tesis doctorales sobre música, semiótica o hibridación cultural. Así recogen, primeramente, su esencia, Romanowsky, George-Warren y Pareles (2001:466), en su famosa 
"Enciclopedia del Rock And Roll", auspiciada por la mítica revista "Rolling Stone":

\author{
“... distinguished of itself from its peers with unusually \\ literate songs (written by Harris) full of hellish imaginery \\ ("Children of the Damned"), their themes borrowed from films \\ ("The Number of the Beast", inspired by The Omen II) and \\ ancient mythology (“Flight of Icarus")... (Rolling Stone, 466)".
}

Por ello no va a ser finalidad de este trabajo retomar en modo alguno la mil veces revisada historia general de la banda liderada por el bajista Steve Harris (Waltham Forest, 1956), que, con 16 discos de estudio y 9 en directo, hasta el momento, se ha consolidado, no ya como un referente de alcance mundial, al margen de la tendencia musical concreta que la alumbró, sino como un irrepetible ejemplo de conexión con el público masivo, previo a la era de internet, cuya fórmula propuso, desde el principio, mixturar la canalización de la rabia del joven europeo de la época (adecuándola a sus intereses formales) con la comunicación y transmisión, tanto del mundo clásico, como de las manifestaciones culturales modernas, preferentemente a través del cine, la Historia, el cómic o la literatura.

Del mismo modo, antes de abordar el objetivo específico de este estudio, cabe destacar que, si bien Iron Maiden ha sido punta de lanza en cuanto a su efectividad práctica sobre las tablas, esa misma esencia pluricultural es la que recorre, sobradamente, la citada "nueva ola" de Heavy Metal británico en la que el grupo se integra, y que, a principios de los años 80 , surgió para dar contestación definitiva, de la mano de grupos igualmente reseñables como Saxon, Def Leppard o Tygers of Pang Tang, a la creciente escena punk que colapsaba el espacio musical juvenil. Tomando algunos de sus elementos estéticos y actitudinales, y filtrándolos a través de un discurso propio, marcado por el fuerte eclecticismo al que aludimos, puede decirse, junto a 
Bonet (1997:21), que "La NWOBHM fue, sin lugar a dudas, el verdadero motor de aquella alternativa musical en toda Europa". Más dispersa, sin embargo, para los analistas, es la precisión del impacto en la juventud de la década de cada uno de los referentes culturales empleados, si bien las crónicas reflejan que, efectivamente, literatura y cine se disputaron abiertamente el pódium. En esta órbita, no se le escapa a Casado (2016:81), en su amplia exégesis sobre la simbología utilizada en la publicidad de este género musical, y su relación con el entorno social, que

"En general, las letras de los temas versan sobre temas variados, leyendas, libros, películas, historia, guerras... Mientras se clasificaba a los aficionados al heavy metal como: 'chicos barriobajeros, ruidosos y sin mucha formación', el hecho de versar las letras sobre temas culturales variados, elevaba el listón cultural de los aficionados".

Martínez Navajas (2015:34), por su parte, precisa mucho más al apostar por la literatura, en su tesis doctoral sobre las "literary covers" del Rock and Roll, cuando incluía un examen detallado sobre la musicalización concreta, por parte de Iron Maiden, del poema mítico de Samuel Taylor Coleridge "The rime of the Ancient Mariner", adaptado en 1984 para el disco "Powerslave":

"Más allá de la frecuente inspiración de las bandas musicales del género en las películas (sobre todo de terror o ciencia ficción), en la mitología o en la Biblia, dentro de estas 'canciones cultas' son las 'versiones literarias' las que más elevan el listón".

No es complicado, sin embargo, encontrar otras fuentes que elevan el cine a un papel preponderante, localizando incluso géneros y subgéneros concretos, dentro del Séptimo Arte, como prima referencia susceptible de versión. Weinstein (2000) integra el "entretenimiento secular" como segunda 
fuente de importancia, dentro de la "retórica e imaginería del caos" que a su entender abraza el heavy, y, tras localizar otro de los ejemplos más recordados de "cover literaria" en los propios Iron Maiden, apunta directamente a las películas de terror, y a las de "Espada y Brujería", como fuentes medulares, las cuales, además, han facilitado con el tiempo la retroalimentación, logrando que las influencias entre cine y música funcionen bilateralmente:

"Heavy metal's second major source of the rethoric and imagery of chaos is secular entertainment. Literature, especially the gothic horror stories of Edgar Allen Poe and the fantasy of H.P. Lovecraft and J.R.R. Tolkien, has inspired songs. Iron Maiden's 'Murders in the Rue Morgue', for example, is a liberal and literal borrowing from Poe's story of that name. Sword and sorcery and horror movies, from Conan the Barbarian to Friday the 13th, have also inspired heavy metal lyrics. Heavy metal's debt to these movie genres, which themselves increasingly use heavy metal songs on their sound tracks, is particulary noticeable on album covers, posters, and stage sets".

En el mismo sentido, e igual que Barron e Inglis (2008:189), cuando apuntan específicamente a las películas de terror ("Indeed, even a cursory exploration of the heavy metal environment reveals a bewildering multiplicity of references to, and from, the horror movie"), se expresa Bayer (2019), extendiendo el fenómeno a la inversa, cuando recuerda que algunos directores han utilizado muy profusamente canciones heavy como fondo extradiegético de sus películas:

"The engagement between metal and cinema has in fact branched out into many filmic áreas and benefitted from various cinematic sub-genres. In one field of productive 


\section{Las nuevas narrativas, en el entorno social \\ Universidad de La Laguna, diciembre de 2019}

interaction, metal music serves as a form of ambient background in horror films that creates an intense and somewhat threatening atmosphere"1.

Pero quizá es Muniesa (1993:45) quien no olvida el hito que supuso que, desde un momento relativamente temprano, la relación entre Heavy Metal y cine cuajase en productos híbridos muy concretos y específicos, que también han hallado su propio recodo en la posteridad:

Como consecuencia del crecimiento de su popularidad, sobre todo en el período 1980-1982, el heavy metal se interrelacionó mucho con otras manifestaciones culturales, básicamente el cine y el cómic (...). Todo el mundo imaginativo del cómic, la ciencia ficción, el futuro de los temas que se trataban con frecuencia en las canciones de los grupos heavys, se desarrolló enormemente, siendo el mejor ejemplo una película, absolutamente emblemática dentro del género, que se estrenó en 1982: 'Heavy Metal, The Movie'”.

\section{Los paisajes cinematográficos de Iron Maiden. Metodología.}

Sobreviviendo a las múltiples formaciones que caracterizaron sus inicios, la banda de Steve Harris estuvo ligada desde el principio al espectáculo visual, contando en sus primeros conciertos con números de ilusionismo en directo, y abrazando una potentísima imagen propia merced a la influencia comiquera de su mascota, Eddie the Head, a la larga puntal indiscutible del éxito y de la imagen de marca del grupo, y considerada un miembro más del mismo

\footnotetext{
${ }^{1}$ Para profundizar en la relación inversa a la que estudiamos en este trabajo, es decir, la influencia de la música heavy en el cine, vale la pena consultar Heavy Metal Movies. Guitar Barbarians, Mutant Bimbos \& Cult Zombies amok in the 666 most ear-and-eye-ripping bigsream films ever! (consultar Bibliografía).
} 
gracias al impacto y fama de su presencia en las portadas de todos sus discos. Como recuerda Christe (2005), sobre las primeras actuaciones de la formación,

\begin{abstract}
“Iron Maiden tomó trucos escénicos shakesperianos como máquinas de humo y elementos decorativos caseros e inquietantes. Aprovechando el entorno, los horrorosos espectáculos de Iron Maiden evocaban típicos terrores británicos tales como las películas de Hammer Films de los sesenta".
\end{abstract}

Sin embargo, una mirada calma a la evolución del grupo, y a su relación con el cine en términos absolutos (más profunda, e inherente, como hemos visto, a la razón de ser de la New Wave), nos permite contradecir algunos de los aspectos expresados hasta ahora. Si bien Christe $^{2}$ (2012), sobre la estética terrorífica (de cinta de terror) que rodea a Maiden desde sus inicios, rememora que

\begin{abstract}
"Alone among that steemed class of 1980, Iron Maiden tapped into the English fog, the cobblestones, and the fear impulses as seen in the gory new tourist attraction London Dungeon, haunted by the shivery ghosts of centuries of monarchy and a few decades of Hammer Horror films",
\end{abstract}

no es menos cierto que la banda, como comprobaremos, sí toma elementos terroríficos de distintos géneros. $O$ al menos les da una apariencia terrorífica, en muchos casos, pero no se vale exclusivamente, ni mucho menos, del cine de terror en exclusiva para configurar su repertorio. El mejor ejemplo de ello es el propio nombre del grupo, extraído de un instrumento de tortura

\footnotetext{
${ }^{2}$ Comentario en Iron Maiden: The ultimate unhautorized history of the Beast (consultar Bibliografía).
} 


\section{Las nuevas narrativas, en el entorno social Universidad de La Laguna, diciembre de 2019}

medieval, es cierto, pero al mismo tiempo imbricado en el imaginario de una indispensable novela de aventuras: "El hombre de la máscara de hierro". Lo recuerda Daniels (2014), en sus textos sobre los orígenes de la formación: "The name of his brainchild would be Iron Maiden, which derives from the movie adaptation of The Man In The Iron Mask, based on the classic Alexandre Dumas novel".

En este sentido, a la creatividad innata de de Steve Harris hay que unir el interesante concepto de "teatro de la mente" en el que el vocalista de Maiden desde 1982, Bruce Dickinson (Worksop, 1958), abunda en su reciente autobiografía “¿Para qué sirve este botón?” 3 , y que se manifiesta progresivamente en su personalidad como artista desde la asunción de parte de la gestualidad dramática sobre el escenario de alguno de sus ídolos, como Ian Anderson, de Jethro Tull, a su querencia particular por los elementos y motivos teatrales presentes, desde siempre, en la identidad del grupo, pasando por el disfrute progresivo de la evolución y educación de sus propias cuerdas vocales. Un cóctel perfecto, a todas luces, para crear esa música tan ciertamente "visual" que se ha convertido, con el paso de las décadas, en fenómeno global y marca de la casa.

Resulta curioso también que, en la biografía oficial del grupo, "Run to the hills. The oficial biography" 4 , cuando se habla pormenorizadamente de los cortes de cada uno de los discos (hasta "Virtual XI", 1998), casi nunca se incide en la inspiración evidente de muchos de ellos en grandes producciones cinematográficas, sino, más bien, de los sentimientos puntuales del compositor de turno a la hora de escribirlos, aunque igualmente evidente sea el hecho de que tales sentimientos se filtran y expresan, en los casos que nos

\footnotetext{
${ }^{3}$ Consultar Bibliografía.

${ }^{4}$ Consultar Bibliografía.
} 
interesan, a través de un motivo de ficción audiovisual. Además, la relación de los miembros de la banda con el cine, del que se consideran amantes declarados, tampoco es parcial ni exclusiva de Steve Harris. En "¿Para qué sirve este botón?", Bruce Dickinson incluye de hecho un momento catártico de su infancia, en concreto su primer contacto con una banda de rock, The Casuals, que tocó cerca de su hogar a finales de los años 60, y con la que coincidió en un pase de "Estación Polar Cebra" (Ice Station Zebra, John Sturges, 1968). Dice el autor (2018:28):

"En el cine Gaumont de Sheffield echaban Estación Polar Cebra. Con palomitas en la mano, diez años, sentado en un cine con una banda de rock viendo una película de guerra sobre submarinos nucleares y misiles, pensé: 'Esto es vida"."

No ha escapado, por último, a la sagacidad de los cronistas, tanta profusión referencial. En múltiples ocasiones se ha preguntado a los miembros de Maiden por esta fructífera simbiosis, siendo, tal vez, Blaze Bailey (Birmingham, 1963), vocalista en los discos "The X Factor" (1995) y el citado "Virtual XI", quien se acerca más a la tesis que propondremos sobre la utilidad de ciertas canciones heavy como bandas sonoras alternativas de algunas películas, ofreciendo una respuesta sorprendente en Popoff (2018:77): la inspiración en el cine y la literatura son vías ideales para evitar la recurrencia excesiva de canciones de amor.

"Well, it's a nice way to avoid love (...). It's interesting to think about the Battle of Britain. It's interesting to think about being back in the village and the questions arising from The prisoner TV show. To think about 'Powerslave' and slavery and being trapped. And 'The Rime of the Ancient Mariner' is a fantastic poem. To take and put it to music and really express that idea, to 
me, that's great. So much of what Maiden has done could be a film soundtrack".

Pese a haber sido perenne objeto de curiosidad, y de miles de comentarios fácilmente rastreables en los anecdotarios de la red, no se habían sistematizado hasta ahora en un trabajo académico las canciones de Iron Maiden basadas en películas, ni estudiado la estructura letrística y musical que ha convertido a cada una en una auténtica "banda sonora" alternativa, capaz de evocar en los aficionados escenas e instantes exactos, si es que han visto el filme en cuestión, cuando no manifiestan la particularidad, casi siempre, de impeler a verlo en caso no haberlo hecho. Todo gracias a las descripciones atmosféricas -en ocasiones realmente cosmogónicas- con las que Iron Maiden transporta al oyente a los mundos de ficción del cine merced a su facilidad y oficio a la hora de construir, con el rico material de base, impresionantes paisajes auditivos.

Analizaremos, por tanto, los temas basados en películas desde su primer disco en estudio, "Iron Maiden" (1980), hasta el último a la hora de la presentación de este trabajo, "The book of souls" (2015), y lo haremos detectándolos, en primera instancia, y analizando después, si procede, determinados pasajes de sus letras, cuando no los más evocadores de la música (es decir, aquellos acordes, arreglos o efectos que sumergen de algún modo en la película), con el objetivo de establecer una pequeña guía de referencia para neófitos y expertos.

Como comprobaremos, y en la línea de lo expuesto más arriba, existen temas en el repertorio de Iron Maiden en los que la línea entre el original y la creación lírica ad hoc de la banda se difuminan, toda vez que se trata de adaptaciones libres, en las que no todo el relato de la canción se corresponde 
con la obra original, o bien éste se anexiona a segmentos autobiográficos puntuales del compositor (como se ha dicho más arriba), o bien dicho relato está basado más en la obra literaria previa que en la adaptación o adaptaciones cinematográficas de la misma, cuando se da el caso de que ambas coexisten. En estos casos, mencionaremos el tema igualmente, aunque no pueda inferirse la correlación total entre el mismo y la obra de cine, conectándolo, eso sí, con las versiones cinematográficas del original cercanas o asimilables.

Obviaremos también, por último, otros elementos del espectáculo tradicional de Iron Maiden relacionados con el cine, como las "intros" de sus actuaciones en directo, en las que, por ejemplo, han llegado a utilizar pasajes de bandas sonoras reales, en los instantes previos a saltar al escenario, como por ejemplo Arthur's Farewell, composición de Jerry Goldsmith para la banda sonora de "El primer caballero" (First Knight, Jerry Zucker, 1995), que pudo escucharse durante la gira titulada Brave New World Tour, en 2002.

\section{Detección y análisis de bandas sonoras "alternativas".}

"Iron Maiden" (1980): tanto este primer larga duración como el segundo, "Killers", abrazan la ecléctica entre punk y heavy, en lo estrictamente musical, que forja la esencia primigenia de la banda, en buena medida gracias a la presencia y voz de su primer vocalista, Paul Di'Anno (Londres, 1958). No hay, acreditada al menos, pista alguna en el listado inspirada en películas concretas, aunque sí una declaración de intenciones en lo tocante a la querencia cultural distintiva de la New Wave. En los años siguientes, temas como su primer corte de rock teatral, "Phantom of the Opera", en torno a la imaginería de la obra de Gaston Leroux, y con versiones cinematográficas de amplia raigambre, como la dirigida por Rupert Julian en 1925 con un inolvidable Lon Chaney, o las sucesivas de 1943 y 1962, abren la vía para la 
evocación y la sinestesia intercultural. El corte, quinto del disco, se inicia con unas notas de guitarra que imitan claramente las que el atormentado protagonista de la obra bien podría haber interpretado al órgano, durante su retiro en las sombras. En ella, además, es un narrador subjetivo quien habla y persigue a un Fantasma al que parece conocer muy bien, con quien tiene pesadillas y sobre el que se permite advertir al oyente: "I've looking so long for you now you won't get away from my grasp./You've been living so long in hiding and hiding behind that false mask (...)./Your looks and your feelings are just the remains of your past (...)./Keep your distance, walk away, don't take his bait (...).

Salvo "Transylvania", canción instrumental con tintes que podrían remitir al Drácula literario o cinematográfico, no hay, en este trabajo inicial de Iron Maiden, insistimos, referencia directa alguna a ninguna película concreta.

"Killers" (1981): dentro aún de la fase de despegue del grupo, de nuevo una única canción, "Murders on the rue Morgue", se basa en un material literario previo trasladado al cine con posterioridad, aunque de nuevo, igualmente, la canción no debe constar en calidad de traslación, o de "novelización" breve, de ninguna versión cinematográfica del relato original de Edgar Allan Poe, sino más bien del relato mismo. Y, en esta línea, aún, se trata de una versión muy libre, toda vez que, durante sus versos acelerados, es de nuevo un narrador subjetivo quien describe y ambienta París, tal y como se sugiere en la obra, aunque al poco tiempo es culpado de los famosos crímenes, que no recuerda haber cometido, de modo que lo que se describe en buena parte es su huida hacia la frontera, perseguido "por toda Francia". El tema apunta finalmente a la autoría del narrador en los asesinatos, cuando manifiesta sorpresivamente que su médico ya le advirtió de que "lo había hecho antes". 
No $\sin$ antes recomendar las versiones cinematográficas del relato, "El fantasma de la Rue Morgue" (Phantom of the Rue Morgue, Roy Del Ruth, 1954), y las dos adaptaciones libres anterior y posterior, "Murders in the Rue Morgue" (Robert Florey, 1932), con Bela Lugosi, y "Murders in the Rue Morgue" (Gordon Hessler, 1971), vale la pena rescatar aquí la "puesta en escena" inicial con que la letra nos adentra en el escenario clásico del relato, por mucho que a la postre se aparte de él: "I remember it as plain as day/Although it happened in the dark of the night/l was strolling through the streets of Paris/And it was cold it was starting to rain./And then I heard a piercing scream./And I rushed to the scene of the crime./But all I found was the butchered remains./Of two girls laid side by side".

"The number of the Beast" (1982): El tercer disco de la banda, considerado por buena parte de la audiencia el mejor aún en nuestros días, habida cuenta de la calidad extrema, y prácticamente sin altibajos, de todos sus temas, supone también el inicio y la confirmación de la relación directa de Iron Maiden con el mundo del cine. Tres de sus ocho canciones se inspiran directamente en el audiovisual (dos en películas y uno en una serie). En concreto, la segunda, "Children of the Damned", en su mayoría de tempo lento, está inspirada en "Los hijos de los malditos" (Children of the damned, Anton Leader, 1964), secuela temática del filme de culto "El pueblo de los malditos" (Village of the damned, Wolf Rilla, 1960), en el que se produce una batalla abierta entre científicos, militares y un puñado de niños cuyos poderes sobrehumanos se daban a conocer en la película original.

Más enjundia, para nuestro estudio, exhibe la tercera canción, The prisoner, basada en la serie británica "El prisionero" (The prisoner, 1967), protagonizada por Patrick McGoohan. El corte no solo relata las cuitas de su protagonista, conocido como "Número 6" y encerrado contra su voluntad en 
un extraño pueblo costero, presumiblemente por su actividad como espía durante la Guerra Fría, sino que además se introduce a los fans con un mítico diálogo extraído de la misma: "-We want information... Information... Information.../-The new number two./-Who is number one?/-You are Number Six./-I am not a number! I am a FREE MAN!/-HAHAHAHAHA!".

El tema comienza tras este pasaje con unos compases lentos, que enseguida se transforman en un riff mucho más veloz, destinado a acrecentar la percepción de una historia de huida. Lo mismo sucede con el solo de guitarra, al que acompañan espaciados dobles golpes de platillo por parte del entonces batería Clive Burr (Londres, 1957), y que abiertamente sugieren los lances en el camino del héroe en fuga. El corte pone el énfasis, no en las razones del confinamiento, que en la serie nunca quedaban claras, sino en la voluntad del prisionero, quien, en sus sucesivos intentos de huida, jamás se quebraba ante las presiones externas. Los versos del estribillo son muy ilustrativos en este sentido: "Not a prisoner, I'm a free man./And my blood is my own now./You'd better scratch me from your black book./Cause' I'll round rings around you".

Por último, el himno indiscutible del disco, y de la banda con el paso de los años, es el tema que da nombre al mismo. "The number of the beast" no solo debe en parte su título a la segunda parte de "La profecía" ("La maldición de Damien", Damien, Omen II, Don Taylor, Mike Hodges, 1978), sino que además el grupo trató de adquirir los servicios de una gran estrella del cine de terror para leer los versos iniciales extraídos del Libro de las Revelaciones. Se cuenta en (Daniels, 2014 :107):

\footnotetext{
"Harris drew inspiration for the title track from the horror film Damien: Omen II and the Robert Burns poem Tam O'Shanter. The band hired actor Barry Clayton to open the song with passages read from the
} 
Book Of Revelation, after Vincent Price requested nothing less than $£ 25,000$ as a fee".

La pieza mezcla durante casi cinco minutos sus propias imágenes pesadillescas con el puro rock and roll que se desprende del solo de guitarra, afirmando además, entre otras cosas, la consagración como vocalista de Bruce Dickinson, cuyo grito entre la estrofa inicial y las subsiguientes, mucho más rápidas, justificó su apodo de juventud como Air raid siren (Sirena de ataque aéreo). Y no solo eso; además cabe añadir cómo, en el videoclip original del tema, la banda se ocupó de incluir extractos de varios clásicos del terror de toda índole, desde cimas del miedo expresionista a manifiestos puros de la serie B. También es Daniels (2014:115) quien lo documenta:

"The original promotional music video features the band playing the song with clips from a number of classic horror films such as Godzilla, War of the Colossal Beast, Crimson Ghost, How to Make a Monster, Nosferatu, The Devil Rides out and The Angry Red Planef'.

"Piece of mind" (1983): En este año, Iron Maiden manifiesta un cambio de registro musical palpable, que no viene dado tan solo por el cambio de batería y la llegada de Nicko McBrain (Hackney, 1952) en sustitución de Clive Burr, sino, en el campo que nos interesa, por el nuevo interés de la banda a la hora de adaptar películas -hasta tres, en este caso, más una referencia lejana en el título de una cuarta- no relacionadas con el género de terror. Se trata, en concreto, de "Where eagles dare", canción que inicia el disco basada en el clásico de cine bélico "El desafío de las águilas" (Where eagles dare, Brian G. Hutton, 1968), a partir de la novela de Alistair McLean; "Quest for fire", a propósito de "En busca del fuego" (La Guerre du feu, Jean Jacques Annaud, 1981), y "To tame a land", sobre la versión de David Lynch de la 
epopeya sci-fi de Frank Herbert "Dune” (Dune, 1984).

Iron Maiden imprimió un ritmo épico a "Where eagles dare", cuyo tema principal de la banda sonora de Ron Goodwin, por cierto, también pudo escucharse como introducción de los directos de la formación en la gira que emprendieron aquel año. La canción es cantada por Bruce Dickinson con largas extensiones vocales al final de los primeros versos de cada estrofa, sugiriendo ecos montañosos, e incluye sonidos de metralletas en los solos de guitarra. Pese a su introducción, que incide también al principio en la descripción del escenario en que se desenvuelve la misión de los héroes interpretados en el cine por Clint Eastwood y Richard Burton, donde realmente hace hincapié es en la misma condición heroica de la gesta. Así lo aspotillan los versos finales: "They dared to go where no one would try/They chose to fly where eagles dare".

De "Quest for fire", la musicalización del penoso viaje de los hombres primitivos en el clásico de Annaud, vale la pena rescatar, ante la carencia de efectos añadidos y la sobriedad general del tema, su ambientación, que Maiden describe con todo lujo de detalles de nuevo en la primera estrofa, para colocarnos a las puertas de la aventura, aunque falsee algunos de los hechos que se cuentan en el filme ("En busca del fuego" es una cinta realista, y por tanto no hace coincidir a hombres con dinosaurios): "In a time when dinosaurs walked the Earth/When the land was swamp and caves were home/In an age when prize posession was fire/To search for landscapes, men would roam". Por último, y sin olvidar que la canción "Die with your boots on" contempla el famoso lema del Viejo Oeste sobre el honor trasladado al cine en 1941 por Raoul Walsh (aunque en este caso no se trata de una adaptación de la película), la de "Dune", que pone fin al álbum, sí supone la más completa abducción del grupo dentro del clásico, tanto literario como 
cinematográfico, de todo el disco, y en buena medida de la historia de Maiden. El corte ha sido definido por (Wall, 1998: 261) como "only comprehensible to readers" de la famosa epopeya, ello a pesar de que no pudo titularse como el original, porque Frank Herbert se negó a ello, rotundamente, en su día.

La condensación de un universo tan amplio obliga aquí a Harris, compositor de la pieza, a permitirse incluir sin complejos toda la terminología ideada por Herbert para sus novelas, confiando en la perspicacia, y en la cultura previa del lector-espectador, a la hora de identificarla. De este modo, palabras familiares para él, como "Fremen", "Muad Dib", "Kwizazt Haderach" o "Gom Jabbar", desfilan abiertamente por un relato aderezado con efectos de naves espaciales, en medio de un sonido con lejanas influencias árabes, que acaso buscan la conexión del receptor con las escenas de los grandes desiertos de Arrakis, planeta donde se centra el grueso de la trama.

"Powerslave" (1984): En pugna -según los expertos e historiadores del rockcon "The number of the Beast", por el número 1 entre los mejores discos de Maiden, el mayor hito de "Powerslave" es la musicalización del poema arriba citado de Taylor Coleridge, pese a que en él caben dos traslaciones de películas, y dos anécdotas cinematográficas al margen, que merece la pena reseñar: el uso extradiegético de "Flash of the blade", contenida en el disco, dentro de una de las escenas de "Phenomena" (Dario Argento, 1985), y el frenético tema "Back in the village", que incluye nuevas referencias a "El prisionero" ("I see sixes all the way", o la propia palabra "Village", que era el nombre que en la serie recibía la prisión). Pero las películas "versionadas" esta vez son "La batalla de Inglaterra" (Battle of Britain, Guy Hamilton, 1969), en el corte inicial del disco, "Aces high", y "Los duelistas" (The duellists, Ridley Scott, 1977), en el tema del mismo nombre. De la primera, que 
traslada la recreación de la contienda aérea en la que la RAF se enfrentó a la Luftwaffe alemana para salvar a Inglaterra de la invasión, es de la única que, por ahora, queda constancia literal, mediante declaraciones de uno de los miembros de la banda, de su valor como banda sonora alternativa. Su ritmo endiablado, y sus ecos en el estribillo, que sugieren con música y palabras los mareantes lances de una batalla en el aire (“Running!/Scrambling!/Flying!”), ha dicho Blaze Bailey, en Popoff (2018:73): "If you've seen the film Battle of Britain with Michael Caine, it really feels like 'Aces high'could've been the soundtrack".

"The duellists", por su parte, es una adaptación libre de la cinta de Ridley Scott, que pone el énfasis en el halo de tradición y trascendencia del acto duelístico, más que en versificar el argumento de la película. Así reza su estribillo: "Oh, oh. Fight for the honour/Fight for the splendor/Fight for the pleasure". La línea de bajo, en este caso, lleva todo el peso de la canción, en la que se produce, a modo de guiño, un duelo de guitarras entre Dave Murray y Adrian Smith, y en la que Dickinson se esfuerza, durante el estribillo, por dar un toque operístico a la acción del relato, que como es sabido se desarrolla en el año 1800.

"Somewhere in time" (1986): Sin extendernos demasiado sobre su celebrada portada, repleta de referencias a Blade runner (Ridley Scott, 1982), y en la que Eddie aparecería, según (Wall, 1998:276) "in his now infamous guise as the mutant offspring of Arnie in The Terminator", "Somewhere in time" es un disco pleno de imaginación y aventuras históricas, que incluyen las exploraciones al Ártico o la vida de Alejandro Magno. La banda, que experimentó en este álbum con guitarras sintetizadas, adapta, esta vez, dos películas en concreto, y conecta lejanamente con una tercera, mediante el título, tal y como hiciera en "Die with your boots on": "Los pasajeros del 
tiempo" (Time after time, Nicholas Meyer, 1979), imbricada en "Caught somewhere in time"; la cima del Free Cinema inspirada en la novela de Alan Sillitoe "La soledad del corredor de fondo" (The loneliness of the long distance runner, Tony Richardson 1962), en el tema del mismo nombre, y "El cielo puede esperar" (Heaven can wait, Warren Beatty, Buck Henry, 1978), también en una pista con el mismo título.

"Caught somewhere in time" es un despliegue de fuerza e intensidad musical, en la que el duelo entre H.G Wells y Jack El Destripador que se refleja en la película no nos es descrito mencionando a ninguno de ellos, sino, de nuevo, mediante las desasosegantes descripciones, de una persecución. En este caso, es el villano quien presumiblemente patrimonializa los versos del tema, animando a su oponente a aceptar una oferta en la que es el alma lo que se pone en juego, mientras repite la frase "Time is always on my side", quizá por cuanto, en la película, El Destripador se consideraba integrado mucho más en la agresiva época contemporánea que en la suya propia. Las notas filtradas por el sintetizador imprimen al tema, de tempo rápido y sofocante, como todos los de Maiden basados en persecuciones y cacerías del hombre, la nota futurista que cabía esperar del concepto del disco, y de la historia que se adapta en particular. En "The loneliness...", por su parte, Maiden se emplea a fondo con sus características "guitarras cabalgantes", tan resolutivas en la recreación de batallas históricas, para trasladarnos la cinética implícita en el acto de correr, y también las sensaciones del corredor, que a veces rayan la angustia, así como los cuestionamientos que éste se hace a sí mismo, tal y como los vivía Colin Smith (Tom Courtenay) en la película de Richardson. "Heaven can wait", por último, toma el título de la cinta de Beatty y Henry, pero se basa exclusivamente en las sensaciones astrales del protagonista, en el umbral entre la vida y la muerte, y en el regreso a su propio cuerpo, cuando, al final, se pregunta si renacerá, si se 
tratará de su verdadera muerte, o si simplemente está soñando. No hay, por tanto, como decíamos, mayor inspiración en el filme referenciado.

"Seventh son of a seventh son" (1988): El primer disco conceptual de la banda, y uno de los más celebrados de su carrera, gira por entero en torno a la adaptación de la serie de novelas de Orson Scott Card iniciada un año antes con "El sépitmo hijo", y, por tanto, no hay canciones inspiradas en películas, aunque el video del tema "Can I play with madness" fue dirigido por el editor habitual del famoso grupo cómico Monty Python, Julian Doyle, pieza clave en películas como "Brazil" (Terry Gilliam, 1985), o "La vida de Brian" (Life of Brian, Terry Jones, 1979). Otro destacado miembro del grupo, Graham Chapman, aparece en el video como el estricto profesor de dibujo que se ve arrastrado a unas cuevas de pesadilla, con la ambientación caótica característica de los filmes de Gilliam. Por último, cabe destacar que Doyle dirigiría en 2008 una película, Chemical Wedding, con Bruce Dickinson como guionista, e incluso con un pequeño papel dentro de la trama.

"No prayer for the dying" (1990): Tres referencias de importancia relacionadas con el cine pueden rastrearse en este disco, del que se desprende cierto estado de transición y de vuelta a los orígenes: la primera, a propósito del primer tema, "Tailgunner", que viene a ser una especie de secuela de "Aces High", y cuyo título ("Artillero de cola") ha relacionado cómicamente Bruce Dickinson con una cinta porno sobre sexo anal. La segunda, "Bring your daughter... to the slaughter", se concibió y fue incluida, en este caso, dentro de una banda sonora real, la de "Pesadilla en Elm Street 5" ( $A$ nightmare on Elm Street 5: The Dream Child, Stephen Hopkins, 1989). Y la última, y más clara, es la referida al tema "Run silent, run deep", escrito a partir de "Torpedo" (Run silent, run deep, Robert Wise, 1958), cinta bélica protagonizada por Clark Gable y Burt Lancaster sobre submarinos en la II 
Guerra Mundial, y en la que pueden escucharse sonidos, en su inicio, similares al viento o a la maquinaria pesada. Musicalmente, estos, y los demás temas del disco, están marcados por cierta simplicidad y sencillez, que se extendieron de hecho a la gira tras tantos años de grandes montajes y efectos especiales, por lo que no se aprecian deseos de experimentar, ni de aderezar los cortes con añadidos periféricos que no sean rock descarnado, como se puede apreciar, sobre todo, en "Bring your daughter...."

"Fear of the dark" (1992): En este disco, último antes de la marcha por unos años de Bruce Dickinson, se recoge un tema dedicado a la serie "El fugitivo" (1963-1967) que un año más tarde conocería versión cinematográfica a cargo de Andrew Davis. Protagonizada por David Janssen, la serie relataba la historia de Richard Kimble, un doctor acusado falsamente de la muerte de su esposa, razón por la que Maiden, una vez más, y como en su musicalización de "El prisionero", pone el acento, en primera persona, en el deseo de justicia y libertad de su protagonista: "I am a fugtive being hunted down like game/l am a fugitive but I've got to clear my name". Curiosamente, esta vez el ritmo no es frenético, ni está salpicado de "guitarras cabalgantes" ni de la velocidad que acompaña a otras historias de persecución, aunque la simple "intro" del tema, con una épica e impresionante línea de percusión, contribuye por sí sola a ilustrar las circunstancias del protagonista.

"The X Factor" (1995): La llegada de Blaze Bailey como vocalista trajo al grupo tres nuevas canciones, en este disco marcado por su tempo lento, inspiradas en películas: "Sign of the Cross", "Man on the edge" $y$ "The edge of darkness". La primera, magníficamente ambientada por unos coros gregorianos iniciales y un ritmo que aúna recogimiento, solemnidad y determinación, aborda solapadamente el divorcio y la crisis profesional de Steve Harris tras la marcha de Dickinson, pero se vale muy abiertamente 
para ello del clásico "El nombre de la rosa" (Le nom de la rose, Jean Jacques Annaud, 1986), creando una composición de 11 minutos, en la que musicalmente caben desde esas sensaciones de miedo del protagonista hasta la descripción de los miembros de la Inquisición. No se escatima además, en el estribillo, la mención directa del original: "The Sign of the Cross/The name of the rose/A fire in the sky/The Sign of the Cross".

"Man on the edge", por su parte, es una clarísima traslación del relato de un hombre al límite planteado por Joel Schumacher en "Un día de furia" (Falling Down, 1993), cuyo título original constituye en sí el estribillo, repetido varias veces, y en la que la música, la única con tempo realmente rápido de todo el disco, traslada al espectador la adrenalina de algunas escenas. Por último, el álbum acoge una formidable musicalización de otro clásico bélico, "Apocalypse Now" (Francis Ford Coppola, 1979), en el que se describe todo el periplo del protagonista desde que le es encomendada la misión de encontrar a su objetivo (un coronel Kurtz al que solo se menciona como "genio" o "poeta" en la canción) hasta la muerte de este último. El tema, inspirado en el título original de la novela de Joseph Conrad, "Heart of darkness", está aderezado con sonidos de helicópteros al principio, y se adentra, como aquella, en los conflictos del corazón humano, siempre sin perder la perspectiva de su personaje principal.

"Virtual Xl" (1998): López (2018) asegura que, pese a que este disco no está entre los favoritos de los fans, precisamente, uno de sus grandes clásicos, “The Clansman", viene inspirado por dos películas de 1995, "Braveheart" (Mel Gibson) y "Rob Roy, la pasión de un rebelde" (Rob Roy, Michael CatonJones), sobre los legendarios líderes guerreros escoceses. Lo cierto es que de la letra se desprende una implicación mayor con la trayectoria de William Wallace, el héroe del primer filme, que con la del segundo, al tiempo que exhibe un lirismo impresionante en cuanto a la recreación musical de la vida 
en las highlands, con aires que empatan con su folclore, y que desembocan en un fenomenal estribillo en el que se grita reiteradamente "Freedom" ("Libertad").

Pese a que "When two worlds collide", el otro tema relacionado con el cine de este disco corto, casi calca el título del clásico de ciencia ficción de Rudolph Maté (When worlds collide, 1951), así como buena parte del relato que en él se cuenta, lo cierto es que algunas crónicas lo han relacionado, también, con cintas más actuales. En Brown (2011) se especifica que este corte habla también, en sentido figurado, sobre choques culturales, aunque, en lo que se refiere específicamente al cine,

"Blaze Bayley told Journalist Sumit Chandra that 'Two Worlds Collide'was 'about the extinction of the human race due to an object coming from outer space and, by sheer coincidence, there are two movies, Armageddon and Deep Impact, that talk about the same thing".

"Brave New World" (2000): Por la puerta grande, e inaugurando, según el consenso general, la "nueva era" de Iron Maiden, el regreso de Bruce Dickinson a la banda en este año desplegó nada menos que cinco nuevas canciones relacionadas con el cine, casi todas adaptaciones directas de la narración que en ellas se aborda, dentro de un disco en el que destacó la trabajadísima producción de Kevin Shirley. El corte que da inicio al disco, "The wicker man", adaptaba la película de culto sobre rituales paganos que Robin Hardy, con guion de Anthony Shaffer, dirigió con el mismo título en 1973 (curiosamente, fueron los mismos autores, al contrario de lo que suele suceder, los que se encargarían a posteriori de la novelización de la obra cinematográfica). Respecto a "The mercenary", es evidente que se dedica a una de las cimas del cine de acción de los 80, "Depredador" (Predator, John McTiernan, 1987), aunque en principio pueda confundirse con alguna de las 
numerosas canciones de temática bélica del grupo. Son versos como "Lose your skin, lose your skull" o "Diablo come again to make trophies out of men" los que evocan al extraterrestre cazador que se presenta en el filme, con ritmo épico y simulador, una vez más, de cacerías humanas. En "The fallen angel", por su parte, la letra pone el énfasis en la desesperación que podía vivir, en este caso el detective John Hobbes (Denzel Washington), en su búsqueda de Azazel, el demoníaco enemigo invisible de "Fallen" (Gregory Hoblit, 1998). Aquí la letra sí nombra al enemigo desde la primera estrofa, apelando a su capacidad para poseer cuerpos: "Azazel is beside you and he's playing a game/Demons are inside you and they're making their play/Watching and they're hiding as they wait for the time/For a devil to get ready and take over your mind". La multiplicidad de coros superpuestos de la voz de Dickinson que se emplean en el tema (como en otros del disco) sugería especialmente aquí, además, la sensación de bilocación y omnipresencia de esta entidad sobrenatural.

La soberbia "The nomad", más tarde, aglutina en sus notas, aunque sin nombrarla directamente, toda vez que se basa en la vida de las tribus guerreras del desierto, el espíritu cinematográfico de la legendaria "Lawrence de Arabia" (Lawrence of Arabia, David Lean, 1962). Así lo especificó Steve Harris, como recoge Brown (2011), al indicar que la canción tenía

"A big Lawrence of Arabia type vibe. That is the sort of stuff I really like, the sort of stuff I get off on -film theme music. It is a big influence really (...). The theme of the music was great and this is what I was talking about before, where the vibe of the music sends us in the right direction for the lyrics. It was definetely one of those sorts of tracks. The vibe was very Middle Eastern and that is what inspired the lyrics".

Como vemos, el propio Harris confirma el hecho de que existen canciones en 
las que, efectivamente, no es necesario adaptar estrictamente el relato cinematográfico que inspira a Maiden en cada momento, sino que es la inspiración misma en los paisajes cinematográficos de su acervo, y su traslación al lenguaje musical, el que a veces trae relatos paralelos, 0 conexos, a las letras que los acompañan.

Situación parecida se produce en el último corte reseñable de este disco, "Out of the silent planet", cuya influencia principal, aunque no total, es la adaptación de "La Tempestad" de Shakespeare que Fred M. Wilcox planteó en el clásico de ciencia ficción "Planeta Prohibido" (Forbidden Planet, 1956), así como la novela de ciencia ficción de C.S. Lewis del mismo nombre. La canción incluye varios cambios de ritmo, aunque merece la pena escuchar su introducción, que remite a los antiguos efectos de sonido que se utilizaban para elucubrar, en el cine de aquella época, cómo sonarían las grandes computadoras del futuro que entonces se imaginaban.

"Dance of Death" (2003): Salvo por su portada, curiosamente la prueba inacabada de un dibujo en principio más ambicioso que a Steve Harris le pareció suficiente, y a la que algunos quisieron hallar similitudes con escenas de la película "Eyes wide shut" (Stanley Kubrick, 1999), tan solo la canción que da título al disco contiene un origen interesante, relacionado con el cine, en este caso nada menos que con el de Ingmar Bergman. De nuevo en Brown (2011), esta vez es el guitarrista Janick Gers (Hartlepool, 1957) quien especifica que la inspiración para el tema llegó a propósito de la escena final de "El séptimo sello" (Det sjunde inseglet, Ingmar Bergman, 1957), en la que un grupo de personas aparece bailando, efectivamente, una "danza de la muerte":

"For a long time I thought of incorporating that image somewhere into the song. Steve took that concept but went 


\section{Las nuevas narrativas, en el entorno social \\ Universidad de La Laguna, diciembre de 2019}

somewhere else with it completely to come up with some amazingly visual lyrics".

Esas "letras visuales" a las que se refiere Gers se despliegan por una pieza extensa, repleta de lírica terrorífica, en la que un hombre cualquiera sufre la experiencia de bailar con los muertos, y que se presenta desde el principio con un ritmo de vals que va in crescendo, reforzando poco a poco las evocaciones desde cualquier ángulo sensorial, hasta el momento en que, hacia la mitad del tema, dicho vals "explota" y se desata, permitiendo casi visualizar a las múltiples figuras de ultratumba bailando alrededor de un protagonista al que, finalmente, dejan ir sin que éste pueda llegar a explicárselo del todo, con el consiguiente sufrimiento que ello le acarrea.

"A matter of life and death" (2006): Este trabajo, por su parte, no contiene canciones basadas en películas, toda vez que, sin ser estrictamente un disco conceptual, como lo fue "Seventh son...", sí está centrado por entero en la temática bélica (sin películas alusivas) y en la religión. El nombre del disco, eso sí, coincide con el de la película de Michael Powell y Emeric Pressburger de 1946 (y "favorita de todos los tiempos"5 de Bruce Dickinson), en la que un aviador británico (David Niven) se enamoraba de la voz femenina que contestaba su mensaje de socorro (Kim Hunter). En España, recordemos, la película se tituló "A vida o muerte", prescindiendo de la traducción original, "Cuestión de vida o muerte", que es la que titula el disco.

"The final frontier" (2010): Dejando aparte la del título, una clara referencia confirmada por Bruce Dickinson de "Star Trek V: La última frontera" (Star Trek V: The final frontier, William Shatner, 1989), este trabajo aborda dos traslaciones musicales claras procedentes del mundo cinematográfico: "El

\footnotetext{
${ }^{5}$ Según refiere en “¿Para qué sirve este botón?
} 


\section{Las nuevas narrativas, en el entorno social Universidad de La Laguna, diciembre de 2019}

hombre que pudo reinar" (The man who would be King, John Huston, 1976), en el tema del mismo nombre, y "Cuando el viento sopla" (When the wind blows, Jimmy T. Murakami, 1986), en "When the wild wind blows". Ambas se extienden durante muchos minutos, plenas de sonidos y reminiscencias muy cercanas al rock progresivo. Así, la primera canción, basada en el texto de Rudyard Kipling y la película de Huston, incide en los aspectos pesimistas de la historia, mientras que, en la versión musical de la triste novela gráfica de Raymond Briggs y posterior película animada de Murakami, el ataque nuclear original de la URSS a la nación británica es sustituido por un terremoto, y la muerte del anciano matrimonio protagonista a causa de la radiación lo es aquí por el suicidio. La composición de Harris, cuya introducción nos permite oír, efectivamente, un fuerte viento soplar, alcanza cotas de emotividad pocas veces vista en la carrera de la banda, por cuanto, igual que en el filme, se detiene minuciosamente en la errónea creencia de los ancianos en todo lo que se les cuenta desde la televisión, por parte de las instancias oficiales, y en todos los años de vida compartidos antes de la catástrofe. Musicalmente, además, el tema llega a tener hasta tres secciones distintas, con lo que la sensación de asistir a la escucha de un relato operístico, o la contemplación de una ficción "cerrada" a través del medio audiovisual, adquiere un refuerzo mucho más evidente y complejo que en otras ocasiones.

"The book of souls" (2015): La última producción de Iron Maiden en el estudio hasta la fecha carece de canciones basadas en películas, aunque sí contiene una balada, "Tears of the clown", dedicada al actor Robin Williams, quien como es sabido se suicidó en 2014. Pese a que las causas se relacionaron con el diagnóstico de una demencia con cuerpos de Levy, Maiden aprovecha para reflexionar en el texto de la canción sobre cuestiones importantes acerca de quienes dedican su vida a entretener a los demás: "Who motivates the motivator?/Facade it has to go./He knows it sooner or later./Smile for 
cameras all ok./But tomorrow is another dat he must get through".

\section{Conclusiones:}

Orson Welles grabó, con el grupo Manowar, unas líneas recitadas para dos de los temas de su disco debut, "Battle Hymns" (1982). Las mismas que grabó, años después, el actor Christopher Lee, quien hasta, muy poco antes de su muerte, en 2015, llegó a realizar sus propias incursiones en el Metal Sinfónico, o a colaborar activamente con bandas del estilo. Cuestiones, sin duda, periféricas al tema de este trabajo, pero que igualmente dan idea de que, en la relación del cine con el rock extremo, existe poco margen para poner en tela de juicio la calidad o el prestigio de sus actores intervinientes. En esta misma órbita, podemos concluir que, en el caso de Iron Maiden, parece reduccionista aplicar el tropo que achaca al género una mayor entrega hacia las películas de terror en exclusiva, por cuanto, del análisis realizado, se extrae una amplia paleta de variedades que tocan, desde el cine "de crítica social" hasta el antiguamente conocido como "de arte y ensayo", la acción, la animación o el cine histórico, con especial querencia, eso sí, por las historias bélicas.

Desde un prisma puramente musicológico, además, las canciones de Iron Maiden inspiradas en películas presentan la cualidad de sumergir al escuchante en atmósferas de una riqueza lírica a caballo entre la traducción del discurso audiovisual, presente en la cinta en cuestión, y la amplificación de la experiencia del visionado más allá de su banda sonora original (con una presencia muy destacada de relatos en los que se producen gestas épicas, persecuciones de algún tipo o cacerías humanas). En este sentido, los efectos especiales añadidos, la intensidad emotiva de las letras (adapten o no por completo el argumento de la ficción), y desde luego la transcripción puntual al pentagrama de las sensaciones y evocaciones que producen las 
películas analizadas, mediante los sonidos vocálicos o de los instrumentos, el tempo general o determinados golpes rítmicos de efecto, justifican la afirmación de que los temas de Iron Maiden constituyen auténticas "bandas sonoras alternativas", por cuanto, en el marco puntual de su estilo heavy, están en disposición de crear, en el espectador, las mismas sensaciones "visuales", en torno al filme escogido, que las extrapolables de su banda sonora original.

\section{Bibliografía:}

L Barron, I Inglis (2008): "Scary Movies, Scary Music, Uses and Unities of Heavy Metal in the Contemporary Horror Films". En VV.AA., Terror tracks. Music, Sound and Horror Cinema. Londres; Equinox Publishing Ltd.

G Bayer (2019): Heavy Metal at the Movies. Florida: CRC Press.

M Bonet (1997): Heavy Metal. Celeste: Madrid.

J Brown (2011): Iron Maiden in the studio: The stories behind every álbum. Chelsea: John Blake Publishing Ltd.

MV Casado Pérez (2016): "Evolución de la simbología utilizada en la comunicación publicitaria del hard rock al metal y su relación con el entorno social" (Tesis Doctoral). Universidad de Valladolid: Facultad de Ciencias Sociales, Jurídicas y de la Comunicación.

N Daniels (2012): Iron Maiden: The ultimate unhautorized history of the Beast. Maryland: Voyageur Press. 
N Daniels (2014): Killers. The origins of Iron Maiden. Glasgow: Bell \& Bain Ltd.

I Christe (2005): El sonido de la Bestia. La Historia del Heavy Metal. Barcelona: Ma non troppo.

B Dickinson (2018): ¿Para qué sirve este botón? Una autobiografía. Barcelona: Libros Cúpula.

A López (2018): Iron Maiden: vida, canciones, simbología, conciertos clave y discografía. Barcelona: Ma non troppo.

JM Martínez Navajas (2015): "La influencia de la literatura fantástica decimonónica en lengua inglesa en el Rock'n Roll. Estudio y análisis de algunas 'literary covers' sobre siete autores del siglo XIX". Facultad de Filología: Universidad Nacional de Educación a Distancia (UNED).

M McPadden (2014): Heavy Metal Movies. Guitar Barbarians, Mutant Bimbos \& Cult Zombies amok in the 666 most ear-and-eye-ripping big-sream films ever! Nueva York: Bazilion Points.

M Muniesa (1993): Historia del Heavy Metal. 25 años de Hard Rock. Madrid: Ediciones VOSA.

J Pareles, P Romanowsy (2001). The Rolling Stone. Enciclopaedia of Rock\&Roll (revised and updated for the 21st century). Fireside: Nueva York.

M Popoff (2018): Iron Maiden: Album by Album. Maryland: Voyageur Press. 
M Wall (1998): Iron Maiden. Run to the hills. The oficial biography. Londres: Sanctuary Publishing Limited.

D Weinstein (2000): Heavy Metal. The Music and its Culture. Boston: Da Capo Press. 
Las nuevas narrativas, en el entorno social

Universidad de La Laguna, diciembre de 2019 